\title{
Ventilator-associated pneumonia caused by multidrug-resistant Gram-negative bacteria: understanding nebulization of aminoglycosides and colistin
}

\author{
J. J. Rouby ${ }^{1 *}\left(\mathbb{0}\right.$, C. Sole-Lleonart ${ }^{2,3}$, J. Rello $0^{4,5,6}$ and the European Investigators Network for Nebulized Antibiotics \\ in Ventilator-associated Pneumonia
}

(c) 2020 Springer-Verlag GmbH Germany, part of Springer Nature

The use of nebulized antibiotics for treating ventilatorassociated pneumonia (VAP) caused by multidrugresistant (MDR) Gram-negative bacteria (GNB) increases worldwide. There is a paradox, however, between the large body of experimental evidence supporting the administration of nebulized rather than intravenous aminoglycosides and colistin to treat inoculation pneumonia caused by GNB [1, 2], and the paucity of clinical studies confirming such a benefit in VAP. Based on the recommendations of the European Society of Clinical Microbiology and Infectious Diseases (ESCMID) [3, 4], the present article examines this apparent contradiction and suggests some directions for further research and clinical practice.

\section{Why and when to administer nebulized aminoglycosides and colistin in VAP}

The main reason for nebulizing aminoglycosides and colistin in VAP is to bypass the alveolar-capillary barrier which offers a severe obstacle to lung penetration following intravenous administration. In comparison to intravenous route, nebulized aminoglycosides and colistin can achieve significantly higher lung tissue concentrations necessary for the effective treatment of VAP due to MDR GNB $[1,2]$. For colistin, this is achieved with minimal

\footnotetext{
*Correspondence: jjrouby@invivo.edu

${ }^{1}$ Multidisciplinary Intensive Care Unit, Department of Anaesthesiology and Critical Care, La Pitié-Salpêtrière Hospital, Assistance Publique Hôpitaux de Paris, Medicine Sorbonne University, Paris, France

Full author information is available at the end of the article
}

systemic toxicity compared to intravenous administration. Demonstration of high lung tissue deposition following nebulization is difficult in humans as epithelial lining fluid concentrations may be in part falsely elevated because of a heavy contamination of the bronchoscope during bronchoalveolar lavage (Fig. 1e-i) [5]. Evidence of high lung tissue concentrations relies on microdialysis [2] or open lung biopsies [1] that can be performed exclusively in experimental studies.

In healthy sheep, high and homogeneously distributed tobramycin pulmonary interstitial concentrations are observed $30 \mathrm{~min}$ after nebulization followed by a bi-compartmental decrease, and contrasting with low concentrations after intravenous administration [2]. In pneumonia, high antibiotic tissue concentrations are also observed but are heterogeneously distributed, and likely influenced by the aeration loss [6,7]. Peak tissue concentrations remain high in non-aerated lung regions, indicating that aminoglycosides and colistin likely diffuse through bronchiolar mucosa towards adjacent consolidated infected alveoli. Both are concentration-dependent antibiotics with peak interstitial concentrations determining bactericidality. Systemic diffusion of nebulized aminoglycosides is substantial, increasing when the alveolar-capillary membrane is injured by a microorganism [1]. The peak plasma concentration is observed $1 \mathrm{~h}$ following nebulisation, with a subsequent bi-compartmental time-dependent decrease. Trough plasma concentrations, which determine the toxicity risk, are similar to those resulting from the intravenous administration when the nebulized dose is equal to the intravenous dose 
plus extrapulmonary deposition (Fig. 1k). In contrast, systemic diffusion of nebulized colistin is weak even in the presence of extensive VAP (Fig. 1j), protecting against nephrotoxicity $[1,7,8]$ and offering the possibility of delivering very high doses by nebulization. Measured colistin plasma concentrations result from the hydrolysis of the nebulized prodrug colistin methanesulfonate [9].

According to available PK data, a benefit of nebulization on cure rate and microbiological eradication can be expected with two classes of antibiotics: aminoglycosides and polymyxins, predominantly used in VAP caused by MDR GNB.

\section{Inhaled substitution rather than adjunctive aminoglycosides or colistin for VAP caused by MDR GNB}

Despite the experimental evidence supporting nebulized antibiotics to treat pneumonia, clinical studies have not shown any mortality benefit when used as adjuvant therapy (nebulized plus intravenous colistin or nebulized aminoglycosides plus intravenous betalactams). However, in VAP caused by MDR GNB, a higher clinical resolution rate was observed with adjuvant therapy [10]. A decrease in the emergence of MDR bacteria was also reported in randomized controlled trials without effect on ventilatorassociated pneumonia relapse [11-13].

The ESCMID position paper [4] recommended avoiding the routine use of nebulized antibiotics in VAP, due to a questionable efficacy and the potential for underestimated risks of adverse respiratory events. The panel identified an urgent need for randomized clinical trials of nebulized antibiotic therapy as part of a substitution approach to VAP therapy caused by MDR pathogens. In 2018, the French Society of Anaesthesia and Intensive Care Medicine (FSAICM) and the French Intensive Care Society (FICS) published guidelines regarding hospital-acquired pneumonia (HAP) in the intensive care unit [11], and recommended nebulized colistin and/or aminoglycosides alone in HAP due to MDR GNB susceptible to colistin and/or aminoglycosides, when no other antibiotics can be used.

When considering experimental pharmacokinetic data, the rationale for adjunctive nebulized therapy appears limited $[4,11]$. The combination of nebulized and intravenous aminoglycosides is likely to increase the risk of toxicity. When added to intravenous betalactams, nebulized aminoglycosides do not improve therapeutic efficacy in VAP caused by susceptible GNB, likely because double-antimicrobial therapy is not superior to monotherapy. The addition of nebulized to intravenous colistin increases lung tissue concentrations but not plasma concentrations. Thus, it improves efficacy without increasing systemic toxicity. Compared to adjunctive therapy, substitution therapy markedly reduces colistin plasma concentrations and decreases the risk of toxicity as shown in a recent meta-analysis [10]. This is the reason why the ESCMID position paper recommended to perform future randomized control studies comparing substitution therapy (rather than adjunctive therapy), to intravenous administration [4]. The FSAICM and FICS also recommended the use of substitution rather than adjunctive therapy in VAP caused by MDR GNB susceptible to colistin and/or aminoglycosides [11].

\section{Optimisation of nebulization to maximize antibiotic lung deposition}

Limiting inspiratory flow velocity is required to reduce inertial impaction in the airways and optimize lung deposition $[1,3]$. Volume-controlled mode should be preferred to pressure support ventilation [14]. As shown in Fig. 1, it is recommended to select specific ventilator settings during the nebulization, to use specifically designed

\footnotetext{
(See figure on next page.)

Fig. 1 Nebulization of amikacin and colistin: from the nebulizer's chamber to lung deposition and urinary excretion. a-c Mesh nebulizers positioned $15 \mathrm{~cm}$ from the $Y$ piece, and made of a domed aperture plate with 1000 precision-formed holes which vibrates at $100 \mathrm{kHz}$. The vibrationinduced micro-pumping effect produces a fine particle, low velocity aerosol. The mass median aerodynamic diameter depends and the holes' diameter; $\mathbf{d}$ specific respirator tubings with smooth angle and inner surface; $\mathbf{e}-\mathbf{i}$ illustrations of tracheobronchial deposition of aerosol particles. Scintigraphic images representing airways and lung deposition of an aerosol of diethylenetriaminepentaacetic acid labeled with technetium-99 m are shown in $\mathbf{e - h}$. Images were obtained in four postoperative neurosurgery patients without pulmonary disease ventilated either in volume-controlled ventilation ( $n=2, \mathbf{e}$ and $\mathbf{f}$ ) or in pressure support ventilation ( $n=2, \mathbf{g}$ and $\mathbf{h}$ ). A part of the aerosol reached the lung periphery, but the majority impacted proximally in the trachea and large bronchi. Lung deposition was significantly greater in patients on volume-controlled mechanical ventilation; whereas, extrapulmonary deposition was significantly lower. i Illustrates the contamination of the bronchoscope during the BAL procedure (the red color indicates high aerosol bronchial concentration). Reproduced from Dugernier et al. [14] and Rouby et al. [5] with the permission of the publishers. $\mathbf{j}, \mathbf{k}$ Illustrate the systemic diffusion of nebulized colistin (j) and aminoglycosides (k) in patients with ventilator-associated tracheobronchitis and in piglets with inoculation pneumonia. Amikacin plasma concentrations after intravenous administration are represented in blue and in black following nebulization. The nebulized dose was equal to the intravenous dose plus extrapulmonary deposition, so that equivalent amount entered the respiratory system either by the trachea (nebulization) or by the pulmonary artery (intravenous administration). Reproduced from Athanassia et al. [8] and Rouby et al. [1] with the permission of the publishers. TV tidal volume, RF respiratory frequency, bpm breaths per minute, I/E inspiratory:expiratory ratio; PEEP positive end-expiratory pressure, BAL bronchoalveolar lavage; $m$ IU million International Units
} 


\section{Nebulized amikacin and colistin for ventilator-associated pneumonia caused by MDR Gram bacteria}
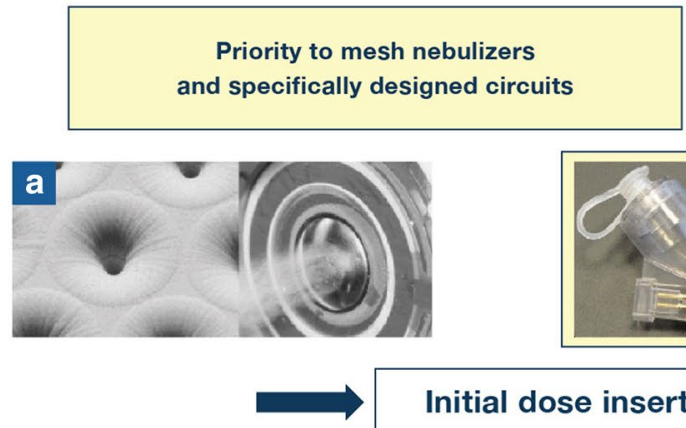

Initial dose inserted into the nebuliser's chamber
Colistimethate $4 \mathrm{~m}$ IU diluted in $6 \mathrm{ml} \mathrm{x3/24h}$

Mesh nebulizers $\longrightarrow$ chamber residual volume $<10 \%$

Jet nebulizers $\longrightarrow$ chamber residual volume $>40 \%$

\section{Amikacin $40 \mathrm{mg} / \mathrm{kg} / 24 \mathrm{~h}$ diluted in $6 \mathrm{ml}$}

Circuits deposit (inspiratory tubing $+\mathrm{Y}$ piece + endotracheal tube) around $\mathbf{3 0 \%}$

Nebulization time $\leq \mathbf{3 0} \mathrm{min}$ - Specific ventilator settings to limit inspiratory inertial impaction

Volume control ventilation with constant inspiratory flow, no patient's triggering. No asynchrony between the patient and the ventilator (propofol if necessary)

TV $8 \mathrm{ml} / \mathrm{kg}$, RF 12-15 bpm, I/E 1:2, end-inspiratory pause $20 \%$, PEEP 5-10 $\mathrm{cmH}_{2} \mathrm{O}$
Removal of heat and moisture exchangers, stop of heat humidifier and addition of a filter on the expiratory limb

Return to previous settings after nebulization Change the expiratory filter

\section{Nebulised dose entering the respiratory system}

Tracheobronchial deposition
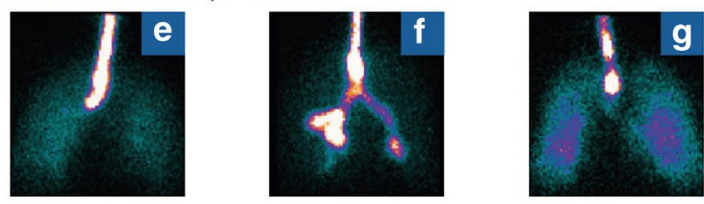

Contamination of the bronchoscope during the BAL

ELF is contaminated during the $\mathrm{BAL}$
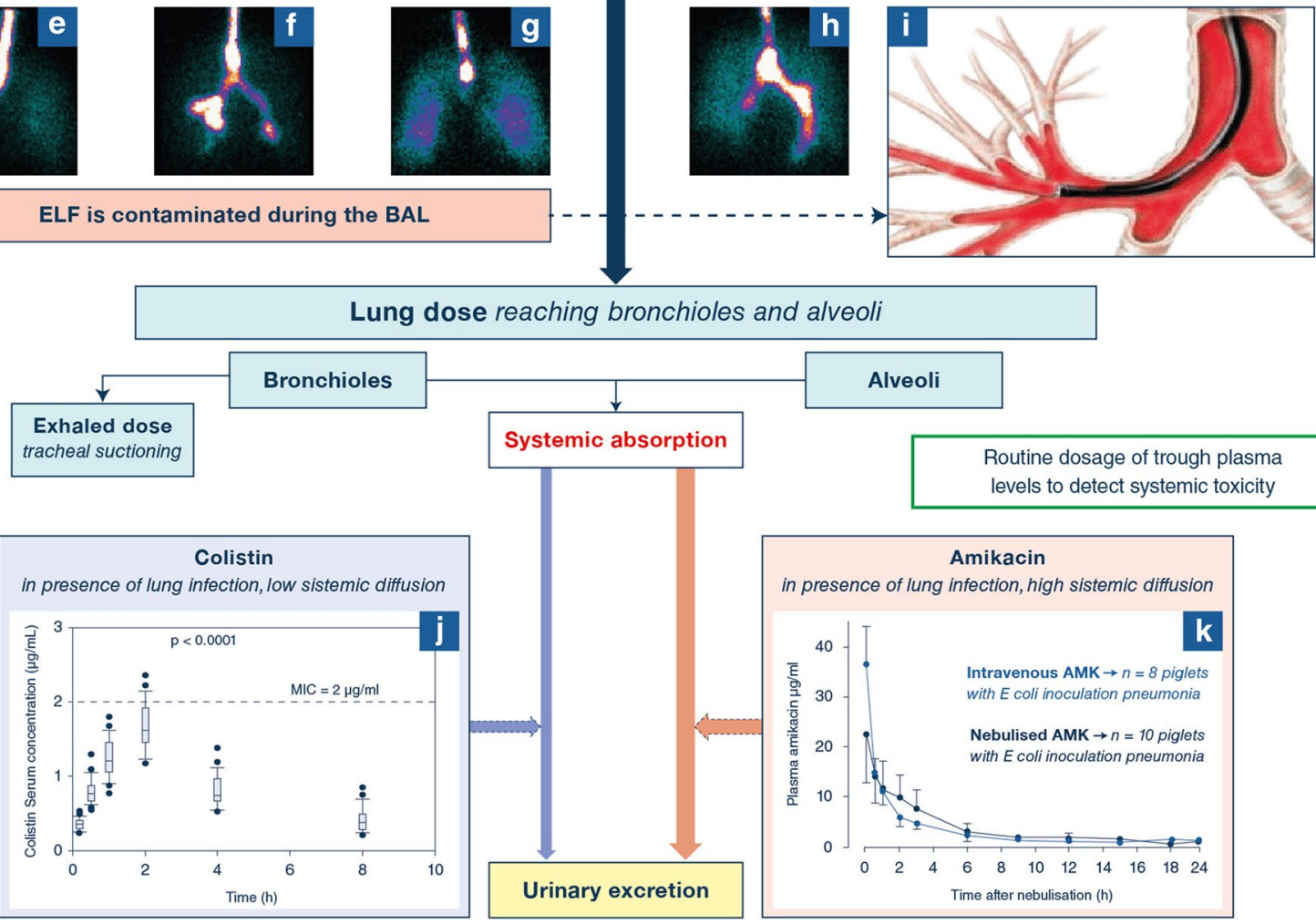

Alveoli

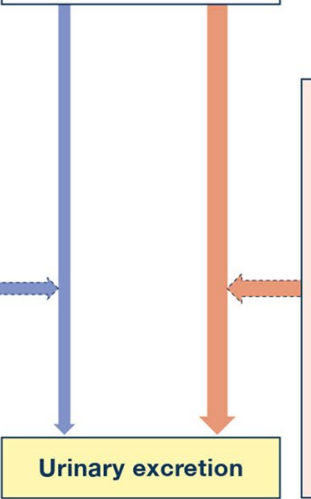

Routine dosage of trough plasma levels to detect systemic toxicity

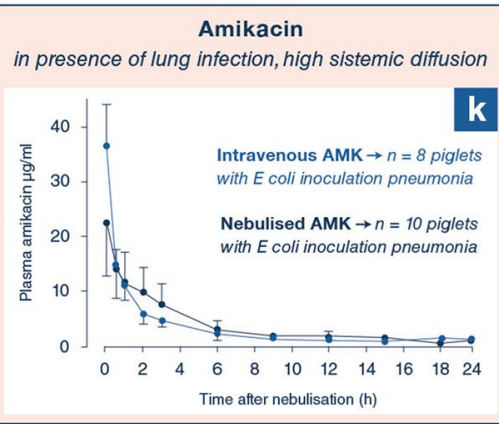


tubings, and mesh nebulizers positioned 10-15 cm before the Y piece on the inspiratory limb [3]. Heat and moisture exchanger and heated humidifiers should be removed during the nebulization to avoid hygroscopic growth of the aerosolized particles and a rainout effect in the circuits. Written operating procedures should be implemented to ensure that previous ventilator settings and humidification are resumed at the end of nebulization. The benefit on aerosol delivery far outweighs the additional workload for health care providers.

There is evidence supporting the use of mesh (Fig. 1a and b) rather than jet nebulizers for nebulized antibiotic delivery $[1,3,11,15]$. In vitro, mesh nebulizers appear superior over jet nebulizers to deliver tobramycin [15]. Aerosol particle size is slightly smaller with jet nebulizers compared to mesh nebulizers, but always remained below five microns, a condition required to reach the distal lung. Lung dose is significantly higher with mesh nebulizers whereas nebulization time and residual volume are significantly reduced. These in vitro benefits were confirmed in animals and in patients treated by salbutamol [16-18].

In conclusion, substitution therapy should be preferred to adjunctive therapy to evidence the therapeutic benefit of nebulized aminoglycosides and colistin in VAP caused by MDR GNB. In addition, mesh nebulizers should be preferred to jet nebulizers to optimize the lung deposition of aerosolized antibiotics.

\footnotetext{
Author details

${ }^{1}$ Multidisciplinary Intensive Care Unit, Department of Anaesthesiology and Critical Care, La Pitié-Salpêtrière Hospital, Assistance Publique Hôpitaux de Paris, Medicine Sorbonne University, Paris, France. ${ }^{2}$ Critical Care Department, Centre Hospitalo-Universtaire Vaudois, Lausanne, Switzerland. ${ }^{3}$ Unitat Cures Intensives, Hospital Universitari de Vic, Barcelona, Spain. ${ }^{4}$ Centro de Investigación Biomédica en Red de Enfermedades Respiratorias (CIBERES), Instituto Salud Carlos III, Madrid, Spain. ${ }^{5}$ Vall d'Hebron Institut of Research (VHIR), Barcelona, Spain. ${ }^{6}$ Anesthesiology Department, CHU Nîmes, Université Nîmes-Montepellier, Nîmes, France.
}

\footnotetext{
Acknowledgements

Members of the European Investigators Network for Nebulized Antibiotics in Ventilator-associated Pneumonia.

Antoine Monsel antoine.monsel@aphp.fr and Jean-Michel Constantin jean-michel.constantin@aphp.fr, Medicine Sorbonne University, Multidisciplinary Intensive Care Unit, Department of Anaesthesiology and Critical Care, La Pitié-Sal pêtrière Hospital, Assistance Publique Hôpitaux de Paris, Paris, France; Adrien Bouglé, adrien.bougle@aphp.fr, Medicine Sorbonne University, Anaesthesiology and Critical Care, Cardiology Institute, Department of Anaesthesiology and Critical Care, La Pitié-Salpêtrière Hospital, Assistance Publique Hôpitaux de Paris, Paris, France; Stijn Blot stijn.blot@UGent.be, Department of Internal Medicine, Faculty of Medicine \& Health Science, Ghent University, Ghent, Belgium; Garyphalia Poulakou gpoulakou@gmail.com Third Department of Medicine, School of Medicine, Sotiria General Hospital, National and Kapodistrian University of Athens, Athens, Greece; Konstantinos Pontikis kostis_pontikis@yahoo.gr, Anna Kyriakoudi Annkyr@gmail.com and Antonia Koutsoukou koutsoukou@yahoo.gr Intensive Care Unit, First Department of Respiratory Medicine, School of Medicine, Sotiria General Hospital, National and Kapodistrian University of Athens, Athens, Greece; George Dimopoulos gdimop@med.uoa.gr Department of Critical Care Medicine, Attikon University
}

Hospital, Medical School, National and Kapodistrian University of Athens, Athens, Greece; Christina Routsi chroutsi@hotmail.com First Department of Intensive Care, School of Medicine, Evangelismos Hospital, National and Kapodistrian University of Athens, Athens, Greece; Kostoula Arvaniti arvanitik@ hotmail.com Papageorgiou Hospital of Thessaloniki, Intensive Care Unit Department, Thessaloniki, Greece; Jose Manuel Pereira jmcrpereira@yahoo.com, Emergency and Intensive Care Department, Centro Hospitalar São João EPE, Faculdade de Medicina da Universidade do Porto, Porto, Portugal; Timothy Felton timothy.felton@manchester.ac.uk, The University of Manchester and Manchester University NHS Foundation Trust, Manchester, United Kingdom; Jayesh Dhanani jadhanani@hotmail.com Burns Trauma and Critical Care Research Centre and Centre for Translational Anti-infective Pharmacodynamics, The University of Queensland, Butterfield Street, Herston, Brisbane, Australia; Jason Roberts j.roberts2@uq.edu.au, (1) University of Queensland Centre for Clinical Research, Faculty of Medicine \& Centre for Translational Anti-infective Pharmacodynamics, School of Pharmacy, The University of Queensland, Brisbane, Australia (2) Departments of Pharmacy and Intensive Care Medicine, Royal Brisbane and Women's Hospital, Brisbane, Australia (3) Division of Anaesthesiology Critical Care Emergency and Pain Medicine, Nîmes University Hospital, University of Montpellier, Nîmes France; Matteo Bassetti matteo. bassetti@asuiud.sanita.fvg.it, Infectious Diseases Clinic, Department of Health Sciences, University of Genoa, Genoa and Hospital Policlinico San MartinoIRCCS, Genoa, Italy; Tobias Welte welte.tobias@mh-hannover.de, University of Hannover, School of Medicine, Hannover, Germany; Jonathan Dugernier Jonathan.dugernier@uclouvain.be and Pierre-François Laterre pierre-francois. laterre@uclouvain.be, St. Luc Clinical Coordinating Center, Department of Critical Care Medicine, St Luc University Hospital, Université Catholique de Louvain Brussels, Belgium; Stephan Ehrmann stephanehrmann@gmail.com, Médecine Intensive Réanimation, CIC INSERM 1415, CRICS-TriggerSep network, CHRU Tours, Tours France and Centre d'étude des pathologies respiratoires INSERM U1100, Université de Tours, Tours, France; Matthieu Boisson matthieu.boisson@chu-poitiers.fr and Olivier Mimoz o.mimoz@chu-poitiers.fr University of Poitiers, Anaesthesiology and Intensive Care Department, University hospital of Poitiers, Poitiers, France; Marc Leone marc.leone@ap-hm.fr University AixMarseille, Department of Anaesthesiology and Critical Care, North Hospital, Marseille, France; Lucy B Palmer lucy.b.palmer@stonybrook.edu Stony Brook University Medical Center Pulmonary, Critical Care and Sleep Division, SUNY at Stony Brook, HSC T17-040, Stony Brook, New York, USA; Jérôme Pugin jerome. pugin@unige.ch Intensive Care Division, University Hospitals of Geneva, Geneva, Switzerland; Jie-ming Qu jmqu0906@163.com (1) Department of Pulmonary and Critical Care Medicine, Rui-jin Hospital, Shanghai Jiao-tong University School of Medicine, Shanghai, China (2) Institute of Respiratory Disease, Shanghai Jiao-tong University School of Medicine, Shanghai, China; Ying-gang Zhu robinzyg@gmail.com Department of Pulmonary and Critical Care Medicine, Hua-dong Hospital, Fudan University, Shanghai, China; Xuelian Liao xuelianliao@hotmail.com, Department of Critical Care Medicine, West China Hospital of Sichuan University, Chengdu, China.

\section{Author contributions}

$J J R$ wrote the first draft of the manuscript. JR and CS-L contributed towards the critical revision of the manuscript for important intellectual content and confirm the integrity of the work. Each member of the European Investigators Network for Nebulized Antibiotics in Ventilator-associated Pneumonia approved the content of the manuscript and contributed to its revision.

\section{Compliance with ethical standards}

\section{Conflicts of interest}

$J R$ received grant support from BAYER and served in the advisory board for BAYER and speakers bureau for Norma Helas. OM served as a consultant for SANOFI. SE declares having received consultancies from Aerogen Ltd, La Diffusion Technique Française and Bayer Healthcare, research support from Aerogen Ltd, Fisher \& Paykel healthcare, Hamilton medical, travel reimbursements from Aerogen Ltd and Fisher \& Paykel. JD and PFL received an unrestricted grant from Aerogen Ltd for their study [14]. JMQ currently received grant from Sino-European Cooperative Clinical Research Project "Evaluation of clinical efficacy and safety of QBW251 in patients with severe bronchiectasis". MB has participated in advisory boards and/or received speaker honoraria from Achaogen, Angelini, Astellas, Bayer, Basilea, Biomerieux, Cidara, Gilead, Menarini, MSD, Nabriva, Paratek, Pfizer, Roche, Melinta, Shionogi, Tetraphase, VenatoRx and Vifor and has received study grants from Angelini, Basilea, 
Astellas, Shionogi, Cidara, Melinta, Gilead, Pfizer and MSD. JAR has received grants or consultancy fees from MSD, Accelerate Diagnostics, Biomerieux, Pfizer, The Medicines Company and Cardeas Pharma. KP has received speaker's Honoraria from MSD and Pfizer. GD has received grants and speaker's Honoraria from Pfizer, InfectoPharma, Virogates, Glenmark india, Clinigen, Nabriva, Menarini and ELPEN. AK has received research grant from Norma Helas. The other authors declare no conflict of interest.

\section{Ethical approval}

An approval by an ethics committee was not applicable.

\section{Publisher's Note}

Springer Nature remains neutral with regard to jurisdictional claims in published maps and institutional affiliations.

Received: 20 October 2019 Accepted: 1 December 2019 Published online: 8 January 2020

\section{References}

1. Rouby JJ, Bouhemad B, Monsel A et al (2012) Aerosolized antibiotics for ventilator-associated pneumonia: lessons from experimental studies. Anesthesiology 117:1364-1380

2. Dhanani JA, Diab S, Chaudhary J et al (2019) Lung pharmacokinetics of tobramycin by intravenous and nebulized dosing in a mechanically ventilated healthy ovine model. Anesthesiology 131:344-355

3. Rello J, Rouby JJ, Sole-Lleonart C et al (2017) Key considerations on nebulization of antimicrobial agents to mechanically ventilated patients. Clin Microbiol Infect 23:640-646

4. Rello J, Solé-Lleonart C, Rouby JJ et al (2017) Use of nebulized antimicrobials for the treatment of respiratory infections in invasively mechanically ventilated adults: a position paper from the European Society of Clinical Microbiology and Infectious Diseases. Clin Microbiol Infect 23:629-639

5. Rouby JJ, Monsel A (2019) Nebulized antibiotics: epithelial lining fluid concentrations overestimate lung tissue concentrations. Anesthesiology 131:229-322

6. Elman M, Goldstein I, Marquette CH, Wallet F, Lenaour G, Rouby JJ (2002) Influence of lung aeration on pulmonary concentrations of nebulized and intravenous amikacin in ventilated piglets with severe bronchopneumonia. Anesthesiology 97:199-206
7. Lu Q, Girardi C, Zhang M et al (2010) Nebulized and intravenous colistin in experimental pneumonia caused by Pseudomonas aeruginosa. Intensive Care Med 36:1147-1155

8. Athanassa ZE, Markantonis SL, Fousteri MZ et al (2012) Pharmacokinetics of inhaled colistimethate sodium (CMS) in mechanically ventilated critically ill patients. Intensive Care Med 38:1779-1786

9. Boisson M, Jacobs M, Grégoire N et al (2014) Comparison of intrapulmonary and systemic pharmacokinetics of colistin methanesulfonate (CMS) and colistin after aerosol delivery and intravenous administration of CMS in critically ill patients. Antimicob Agents Chemother 58:7331-7339

10. Solé-Lleonart C, Rouby JJ, Blot S et al (2017) Nebulization of antiinfective agents in invasively mechanically ventilated adults: a systematic review and meta-analysis. Anesthesiology 126:890-908

11. Leone M, Bouadma L, Bouhemad B et al (2018) Hospital-acquired pneumonia in ICU. Anaesth Crit Care Pain Med 37:83-98

12. Kollef MH, Ricard JD, Roux D et al (2017) A randomized trial of the amikacin fosfomycin inhalation system for the adjunctive therapy of Gram-negative ventilator-associated pneumonia: aFIS trial. Chest 151:1239-1246

13. Lu Q, Yang J, Liu Z, Gutierrez C, Aymard G, Rouby JJ, the Nebulized Antibiotics Study Group (2011) Nebulized ceftazidime and amikacin in ventilator-associated pneumonia caused by Pseudomonas aeruginosa. Am J Respir Crit Care Med 184:106-115

14. Dugernier J, Reychler G, Wittebole X et al (2016) Aerosol delivery with two ventilation modes during mechanical ventilation: a randomized study. Ann Intensive Care 6(1):73

15. Mashat M, Clark BJ, Assi KH, Chrystyn H (2016) In vitro aerodynamic characterization of the dose emitted during nebulization of tobramycin high strength solution by novel and jet nebulizer delivery systems. Pulm Pharmacol Ther 37:37e42

16. Chang KH, Moon SH, Oh JY et al (2019) Comparison of salbutamol delivery efficiency for jet versus mesh nebulizer using mice. Pharmaceutics 11:192. https://doi.org/10.3390/pharmaceutics11040192

17. Galindo-Filho VC, Alcoforado L, Rattes C et al (2019) A mesh nebulizer is more effective than jet nebulizer to nebulize bronchodilators during noninvasive ventilation of subjects with COPD: a randomized controlled trial with radiolabeled aerosols. Respir Med 153:60-67

18. Dunne RB, Shortt S (2018) Comparison of bronchodilator administration with vibrating mesh nebulizer and standard jet nebulizer in the emergency department. Am J Emerg Med 36:641-646 